\title{
Hallazgos ecográficos en la proctalgia espontánea y postoperatoria
}

\author{
I. Pascual, D. García-Olmoํㅡ, C. Martínez-Puente y J. A. Pascual-Montero \\ Clínica Cemtro. ${ }^{1}$ Universidad Autónoma. Madrid
}

\section{RESUMEN}

Objetivo: valorar la utilidad de la ecografía endoanal como prueba de imagen para identificar la causa del dolor anal en los pacientes que presentan proctalgia idiopática o dolor postoperatorio y analizar cuáles son sus causas más frecuentes.

Métodos: se realiza un estudio descriptivo de los hallazgos encontrados en las ecografías endoanales de pacientes con dolor anal en los últimos seis años. Todas las ecografías se llevaron a cabo con un ecógrafo B\&K (Cheetah 2003, B\&K Medical, Gentofte, Denmark) con sonda endoanal de $7 \mathrm{MHz}$.

Resultados: se estudiaron noventa y cinco casos de proctalgia mediante ecografía endoanal. Sesenta y siete correspondieron a pacientes con una cirugía previa perineal o pélvica tras la cual comenzó el dolor anal: 48 habían sido intervenidos de fisura anal, 12 de hemorroidectomía, 4 de episiotomía, 2 de fístula y 1 de prostatectomía. El hallazgo más frecuente tras la cirugía de fisura anal fue la presencia de una esfinterotomía incompleta. Entre los veintiocho pacientes sin cirugía previa, el 57,14\% presentaba hipertrofia del esfínter anal interno como única alteración ecográfica.

Conclusiones: los pacientes con proctalgia espontánea y postoperatoria pueden ser estudiados mediante ecografía endoanal ya que el uso de la sonda no impide completar la exploración. Con esta prueba se encontró una causa del dolor en el 81,93\% de los casos. La hipertrofia del esfínter anal interno aislada es el hallazgo ecográfico más frecuente asociado a proctalgia espontánea.

Palabras clave: Ecografía endoanal. Fisura anal. Proctalgia.

\begin{abstract}
Objective: to assess the use of endoanal ultrasounds to identify anal pain etiology in patients with either spontaneous or postoperative pain, and to review the most frequent causes.

Methods: a descriptive study of ultrasound findings in patients with anal pain during the last six years was performed. All ultrasound scans were performed using a B\&K Diagnostic Ultrasound System (Cheetah 2003, B\&K Medical, Gentofte, Denmark) with a 7-MHz endoprobe providing $360^{\circ}$ images.

Results: ninety-five cases of anal pain were studied by endoanal ultrasonography. Sixty-seven cases of anal pain ocurred in patients with previous perineal or pelvic surgery: anal fissure (48), hemorrhoidectomy (12), episiotomy (4), fistula (2), and prostatectomy (1). After fissure surgery, incomplete sphincterotomy was the first cause of anal pain. Twenty-eight patients had no previous surgery, and more than $57.14 \%$ of them were found to have internal anal sphincter hypertrophy.

Conclusions: patients with anal pain can be studied by endoanal ultrasounds in spite of the use of an endoprobe. With this exploration a cause of pain is found in $81.93 \%$ of cases. Internal anal sphincter hypertrophy is the most frequent finding associated with spontaneous anal pain.
\end{abstract}

Key words: Endoanal ultrasounds. Fissure. Anal pain.

Pascual I, García-Olmo D, Martínez-Puente C, Pascual-Montero JA. Hallazgos ecográficos en la proctalgia espontánea y postoperatoria. Rev Esp Enferm Dig 2008; 100: 764-767.

Recibido: $15-07-08$.

Aceptado: 10-10-08.

Correspondencia: Isabel Pascual. Servicio de Cirugía General. Coloproctología. Clínica Cemtro. Avda. Ventisquero de la Condesa, 42. 28035 Madrid.e-mail: isabelpasmi@ hotmail.com

\section{INTRODUCCIÓN}

El dolor anal es un síntoma muy frecuente en toda la patología anorrectal. En la mayoría de los casos se asocia a un proceso orgánico fácilmente reconocible mediante una cuidadosa exploración física (fisura, trombosis hemorroidal, absceso perianal, etc.). En pocas ocasiones es 
necesario recurrir a una prueba de imagen para establecer la causa del dolor.

Sin embargo, hay casos de proctalgia que requieren un estudio más profundo para conocer la etiología del dolor y poder ofrecer un tratamiento adecuado a cada paciente. El dolor postoperatorio que se prolonga en el tiempo tras una cirugía proctológica hace necesario establecer un diagnóstico mediante una prueba de imagen para poder resolverlo. Los pacientes con dolor espontáneo y sin patología en la exploración física también requieren un estudio más exhaustivo de dolor, ya sea mediante ecografía endoanal y/o endoscopia.

Aunque la ecografía endoanal ha sido ampliamente utilizada en la evaluación de diversas patologías anorrectales como la incontinencia, las fístulas perianales y la estadificación del cáncer de recto (1-5), su uso en los casos de dolor ha sido muy limitado. Esto podría deberse a dos razones: por una parte, a la escasez de casos en los que la causa del dolor no se diagnostica mediante el examen físico; y por otro lado, a la necesidad de usar una sonda endoanal para realizar este tipo de ecografía que podría exacerbar el dolor. En este estudio valoramos la factibilidad y la utilidad de la ecografía endoanal en los casos de proctalgia espontánea y postoperatoria.

\section{MÉTODOS}

Se realiza un estudio descriptivo de los hallazgos encontrados en las ecografías endoanales llevadas a cabo en nuestra unidad en los últimos seis años en pacientes con:

- Proctalgia después de una intervención quirúrgica que se prolonga en el tiempo más de lo habitual (a partir de la octava semana postoperatoria) y sin diagnóstico mediante exploración física cuidadosa en consulta.

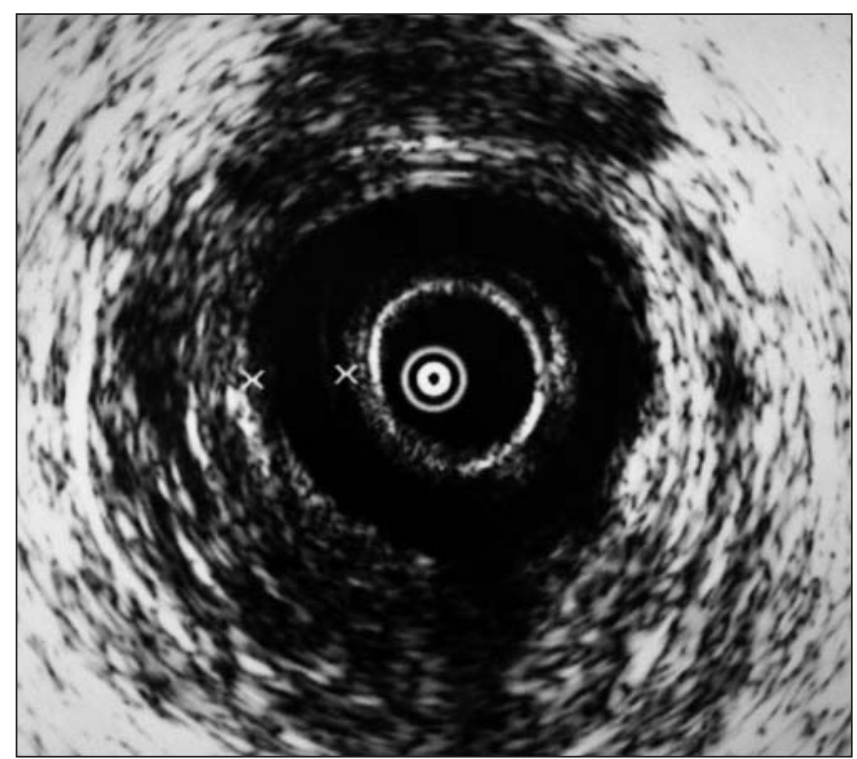

Fig. 1. Esfínter anal interno hipertrófico y simétrico en toda la circunferencia medido en la unión de intercuadrantes derechos.

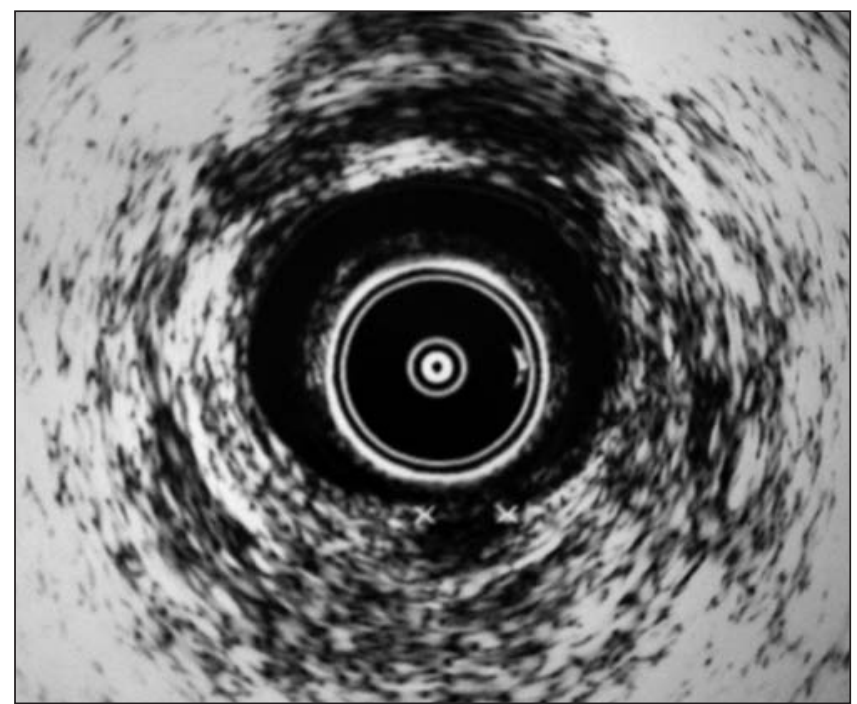

Fig. 2. Esfínter anal interno con esfinterotomía incompleta posterior y área hipoecoica subyacente.

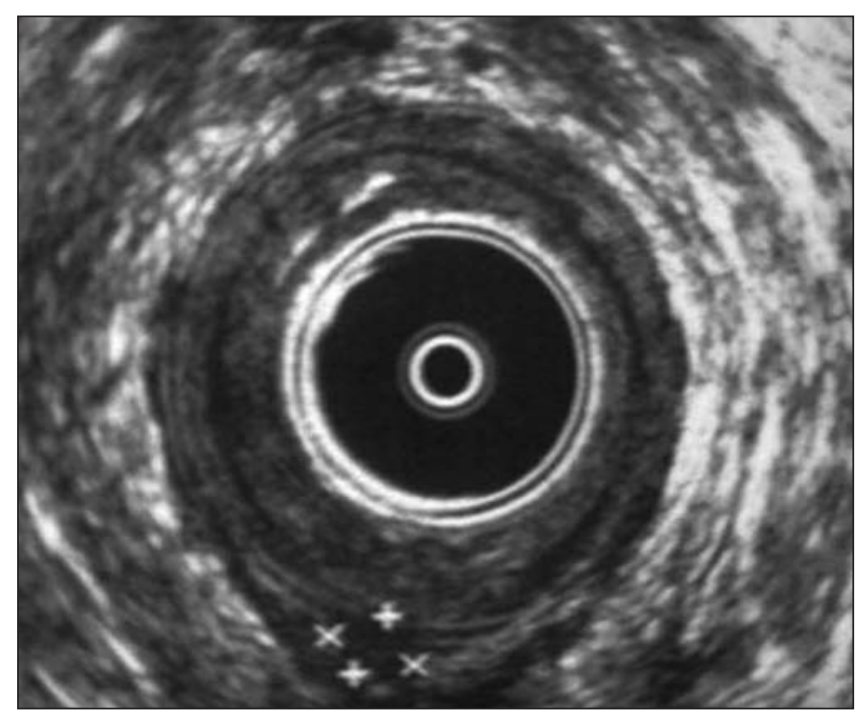

Fig. 3. En posición posterior y lateralizado hacia la derecha destaca una imagen hipoecoica, transmural que rebasa el puborrectal de $2 \mathrm{~cm}$ de diámetro compatible con hematoma de pared tras hemorroidectomía mediante técnica de Longo.

- Proctalgia espontánea de etiología desconocida, sin hallazgos en la exploración física que justifiquen el dolor anal.

Todas las ecografías fueron realizadas en la consulta por cirujanos generales con especial dedicación a la coloproctología con un ecógrafo B\&K (Cheetah 2003, B\&K Medical, Gentofte, Denmark) con sonda endoanal de $7 \mathrm{MHz}$

El protocolo seguido en cada exploración, tras explicar al paciente en qué consistía la prueba, fue la identificación de los tres planos ecográficos del canal anal (superior, medio e inferior) y el análisis posterior de los hallazgos en cada uno de ellos. Consideramos que existe hipertrofia del esfínter anal interno cuando este mide más 
de $3 \mathrm{~mm}$ (6). Describimos como cavidad una área hipoecoica, anfractuosa, a veces con ecos en su interior y heterogénea, que generalmente se encuentra en continuidad con la mucosa en algún punto y cuyo diagnóstico de sospecha es un absceso. Y hablamos de nódulos hipoecoicos para referirnos a aquellas formaciones de baja ecogenicidad, redondeadas y homogéneas que pudieran corresponder a hematomas o cicatrices.

\section{RESULTADOS}

Se revisaron noventa y cinco casos de dolor anal: sesenta y siete correspondieron a pacientes con cirugía perineal o pélvica previa tras la cual había comenzado el dolor (Tabla I) y veintiocho a pacientes con dolor espontáneo (Tabla II). Cincuenta y dos pacientes eran hombres y cuarenta y tres mujeres, con una edad media de 43 años.

Tabla I. Pacientes con dolor anal postoperatorio

\begin{tabular}{llc}
\hline Tipo de cirugía & Hallazgos ecográficos & Número $(\%)$ \\
\hline Fisura (48) & Esfinterotomía incompleta & $17(25,37)$ \\
& Esfinterotomía incompleta & $13(19,40)$ \\
& con EAl hipertrófico & $6(8,95)$ \\
& Cavidad subfisuraria & $5(7,46)$ \\
& Nódulo hipoecoico & $7(10,44)$ \\
\hline Sin hallazgos & \\
\hline Hemorroidectomía Milligan & Nódulo hipoecoico & $3(4,47)$ \\
Morgan (7) & EAl hipertrófico & $2(2,98)$ \\
& Sin hallazgos (EAl adelgazado) & $2(2,98)$ \\
\hline Hemorroidectomía Longo (5) & Sin hallazgos & $4(5,97)$ \\
& Hematoma & $1(1,49)$ \\
\hline Episiotomía (4) & Sin hallazgos & $2(2,98)$ \\
& Fisura & $2(2,98)$ \\
\hline Fístula (2) & Sin hallazgos & $1(1,49)$ \\
& Nódulo hipoecoico & $1(1,49)$ \\
\hline Prostatectomía (1) & Sin hallazgos & $1(1,49)$ \\
\hline Total & & 67 \\
\hline EAl: esfnos
\end{tabular}

EAl: esfínter anal interno. Si en la ecografía sólo se encuentran cambios postoperatorios, sin poder identificar una causa del dolor, esta se clasifica como "sin hallazgos".

Tabla II. Pacientes con proctalgia espontánea

\begin{tabular}{lc}
\hline Diagnóstico & Número $(\%)$ \\
\hline EAl hipertrófico & $16(57,14)$ \\
Cavidad compatible con absceso & $7(25)$ \\
Coxigodinia & $2(7,14)$ \\
Sin hallazgos & $3(10,71)$ \\
Total & 28 \\
\hline
\end{tabular}

El hallazgo más frecuente entre los pacientes operados de fisura anal fue la presencia de una esfinterotomía incompleta, lo que supone un $62,5 \%$ de los pacientes con dolor anal tras cirugía de fisura. El esfínter anal interno se encontraba adelgazado en algún punto del anillo esfinteriano pero completo en treinta casos. En trece de ellos además se encontró un esfínter anal interno hipertrófico -mayor de $3 \mathrm{~mm}$ - (3). En seis pacientes intervenidos de fisura se encontraron cavidades subfisurarias y en cinco nódulos hipoecoicos que podrían corresponder a hematomas o cicatrices en la zona de la esfinterotomía. En siete pacientes no se encontraron hallazgos que justificaran el dolor.

De los doce pacientes intervenidos previamente de hemorroidectomía, en siete se había realizado un procedimiento de Milligan Morgan y en cinco la técnica de Longo. Entre los primeros, tres tenían nódulos hipoecoicos, dos hipertrofia del esfínter anal interno y en dos no se encontró nada que justificase el dolor (aunque se observó un esfínter anal interno adelgazado en ambos casos). En un paciente del segundo grupo, en los que se había realizado la técnica de Longo, se identificó un hematoma en el espesor de la pared rectal.

De los cuatro pacientes con episiotomía previa, en dos se encontró una fisura anal como causa del dolor y en otros dos no hubo hallazgos en la ecografía que justificasen el dolor postoperatorio. En los dos pacientes operados de fístula perianal, uno presentaba un nódulo hipoecoico (compatible con hematoma o cicatriz) y en el otro no se evidenció la causa del dolor. En el paciente operado de prostatectomía sólo se observaron cambios postoperatorios, sin encontrar una causa de proctalgia.

En el grupo de veintiocho pacientes con dolor espontáneo, dieciséis presentaron hipertrofia del esfínter anal interno, siete imágenes compatibles con abscesos, dos coxigodinia y tres tenían una exploración normal.

\section{DISCUSIÓN}

La ecografía endoanal ha demostrado ser, en la serie analizada, una prueba factible y eficaz en el diagnóstico de los casos de proctalgia seleccionados. Todos los pacientes remitidos por dolor anal aceptaron la realización de la prueba una vez informados, y esta se pudo completar sin incidencias. En el 81,93\% de los casos se ha encontrado una causa ecográfica del dolor.

La mayoría de los pacientes con dolor anal postoperatorio habían sido operados de fisura anal, y el hallazgo más frecuente en este grupo de pacientes fue la presencia de una esfinterotomía incompleta. La cirugía estándar de la fisura anal es hoy en día la esfinterotomía lateral interna (ELI). A pesar de ser una intervención relativamente frecuente y sencilla de realizar, a veces es difícil encontrar el equilibrio en este tipo de cirugía. El éxito de la ELI se basa en saber cuáles son la profundidad y la longitud adecuadas en la sección del esfínter para disminuir la hipertonía y curar la fisura sin producir incontinencia. Cuando no se dispone de datos manométricos y/o ecográficos previos, la decisión de dónde y cuánto músculo cor- 
tar se realiza durante la cirugía según los hallazgos encontrados y la experiencia personal del cirujano. Por eso nos parece aconsejable la realización de manometría preoperatoria en los pacientes que vayan a ser intervenidos de fisura anal y ecografía endoanal en todos aquellos que continúen con dolor tras una intervención de fisura anal para poder orientar una reintervención. Así podremos distinguir entre un grupo de pacientes con esfínter normal de aquellos con hipertrofia esfinteriana. Los últimos necesitarán un corte más profundo para asegurar una esfinterotomía completa y el alivio del dolor (7).

Cuando encontramos cavidades o abscesos subfisurarios tras una esfinterotomía lateral interna por fisura anal, nos planteamos si en algunos pacientes es necesario asociar a la esfinterotomía lateral interna la extirpación de la fisura. Este gesto es muy discutido, porque con la sección del esfínter desaparece la hipertonía y la fisura remite en la mayoría de los casos $(8,9)$. Sin embargo, en nuestro grupo creemos que las fisuras crónicas responden mejor si se extirpan y se legran las cavidades subfisurarias, ya que estas permanecen en muchas ocasiones a pesar de realizar una esfinterotomía completa y son causa de dolor crónico postoperatorio, como hemos visto en algunos casos de esta serie $(10,11)$.

Más del $50 \%$ de los pacientes con dolor anal espontáneo tenían hipertrofia del esfínter anal interno como único hallazgo ecográfico. Existe un tipo de hipertrofia esfinteriana hereditaria que cursa con dolor anal compatible con proctalgia fugax y estreñimiento denominada miopatía hipertrófica del esfínter anal interno. Para confirmar el diagnóstico es necesaria una biopsia que demuestre vacuolización de las células musculares lisas (12-15). En los pacientes con hipertrofia esfinteriana que no respondan a tratamiento médico habría que descartar esta patología, ya que su tratamiento es quirúrgico, mediante esfinterotomía lateral interna.

En los casos de dolor anal en los que no se encuentren hallazgos en la ecografía endoanorrectal y se haya excluido cualquier patología orgánica mediante exploración física y endoscópica, debe valorarse el diagnóstico de dolor anorrectal funcional (16). Este síndrome tiene una prevalencia en la población general relativamente alta que se estima entre 6,6 y $14 \%(17,18)$, y se han descrito dos tipos de dolor: la proctalgia fugax y la proctalgia crónica. La exclusión de patología orgánica y la adecuación del tipo de dolor a los criterios descritos en la literatura resultan imprescindibles para el diagnóstico de dolor funcional (19).

La ecografía endoanal ha resultado tener una alta rentabilidad diagnóstica en los pacientes con dolor anal. $\mathrm{Su}$ eficacia y su disponibilidad en la consulta la convierten en la primera prueba a realizar en casos de proctalgia de diagnóstico incierto, tanto espontánea como postoperatoria. Sus resultados permiten orientar una reintervención, realizar un tratamiento conservador en casos de hematomas o cicatrices y descartar patología orgánica en los casos de sospecha de dolor anorectal funcional. La hipertrofia del esfínter anal interno aislada ha resultado ser la causa más frecuente de dolor anal y habrá que valorar en cada caso la opción terapéutica más adecuada.

\section{BIBLIOGRAFÍA}

1. Saranovic D, Barisic G, Krivokapic Z, Masulovic D, Djuric-Stefanovic A. Endoanal ultrasound evaluation of anorectal diseases and disorders: technique, indications, results and limitations. Eur J Radiol 2007; 61: 480-9.

2. Pascual Miguelañez I, García Olmo D, Martínez Puente MC, Pascual Montero JA. Is routine endoanal ultrasound useful in anal fistulas? Rev Esp Enferm Dig 2005; 97: 323-7.

3. De la Portilla F, León-Jiménez E, Cisneros N, Rada R, Flikier B, Vega J, et al. Use of anorectal ultrasounds in perianal Crohn's disease: consistency with clinical data. Rev Esp Enferm Dig 2006; 98(10): 747-54.

4. Fernández-Frías AM, Pérez-Vicente F, Arroyo A, Sánchez-Romero AM, Navarro JM, Serrano P, et al. Is anal endosonography useful in the study of recurrent complex fistula-in-ano? Rev Esp Enferm Dig 2006; 98(8): 573-81.

5. Vila JJ, Jiménez FJ, Irisarri R, Martínez A, Amorena E, Borda F. Rectal cancer staging with endoscopic ultrasonography: correlation with pathological staging. Rev Esp Enferm Dig 2007; 99(3): 132-7.

6. Stoker J, Halligan S, Bartram C. Pelvic floor imaging: state of the art. Radiology 2001; 218: 621-41.

7. Rosa G, Lolli P, Piccinelli D, Mazzola F, Zugni C, Ballarin A, et al. Calibrated lateral internal sphincterotomy for chronic anal fissure. Tech Coloproctol 2005; 9: 127-31.

8. Sánchez Romero A, Arroyo Sebastián A, Pérez Vicente F, Serrano Paz P, Candela Polo F, Tomas Gómez A, et al. Open lateral internal anal sphincterotomy under local anesthesia as the gold standard in the treatment of chronic anal fissures. A prospective clinical and manometric study. Rev Esp Enferm Dig 2004; 96: 856-63.

9. Tocchi A, Mazzoni G, Miccini M, Cassini D, Bettelli E, Brozzetti S. Total lateral sphincterotomy for anal fissure. Int $\mathbf{J}$ Colorectal Dis 2004; 19: 245-9.

10. Pelta AE, Davis KG, Armstrong DN. Subcutaneous fissurotomy: a novel procedure for chronic fissure-in-ano. A review of 109 cases. Dis Colon Rectum 2007; 50(10): 1662-7.

11. Aigner F, Conrad F. Fissurectomy for treatment of chronic anal fissures. Dis Colon Rectum 2008; 51(7): 1163.

12. Martin JE, Swash M, Kamm MA. Myopathy of internal anal sphincter with polyglucosan inclusions. J Pathol 1990; 161: 221-6.

13. Kamm MA, Hoyle CH, Burleigh DE. Hereditary internal anal sphincter myopathy causing proctalgia fugax and constipation. A newly identified condition. Gastroenterology 1991; 100: 805-10.

14. De la Portilla F, Borrero JJ, Rafel E. Hereditary vacuolar internal anal sphincter myopathy causing proctalgia fugax and constipation: a new case contribution. Eur J Gastroenterol Hepatol 2005; 17: 359-61.

15. Martorell P, Azpiroz F, Malagelada JR. Hypertrophic myopathy of the internal anal sphincter: a rarely recognized cause of proctalgia. Rev Esp Enferm Dig 2005; 97: 527-9.

16. Whitehead WE, Wald A, Diamant NE, Enck P, Pemberton JH, Rao SS. Functional disorders of the anus and rectum. Gut 1999; 45: 55-9.

17. Drossman DA, Li Z, Andruzzi E. U.S. Householder survey of functional gastrointestinal disorders: Prevalence, sociodemography, and health impact. Dig Dis Sci 1993; 38: 1569-80.

18. Wald A. Anorectal and pelvic pain in women: diagnostic considerations and treatment. J Clin Gastroenterol 2001; 33(4): 283-8.

19. Bharucha AE, Wald A, Enck P, Rao S. Functional anorectal disorders. Gastroenterology 2006; 130: 1510-8. 